Tôhoku Math. Journ.

32 (1980), 505-510.

\title{
NOTES ON THE STABILITY IN VARIATION
}

\author{
Dedicated to Professor Taro Yoshizawa on his sixtieth birthday
}

JUNJi Kato AND SADAO KUROSAKI

(Received June 21, 1979)

For linear differential equations it is obvious that the stability of an arbitrary solution is equivalent to the stability of the zero solution. However, the situation will be quite different for nonlinear equations. Such a problem on the stability of each of solutions was posed and discussed by several authors as extreme stability of the system [5], [10], (positively) equi-continuous flow [8], stability in variation [2], [3], [6], [9], and so on.

To study perturbation problems of nonlinear differential equations, Alekseev's formula of constant variations [1] presents a useful approach, and it is still more powerful if one assumes some sorts of stability in variation. (cf. [2], [3], [6], [7], [9].) On the other hand, it is also wellknown that Liapunov's second method is an effective tool for perturbation problems (see [10]). Leela [6] and Kamala-Lakshmikantham [3] gave a way to construct desirable Liapunov functions under the stability in variation. However, there was an inaccuracy in their proof, as we see in Remark 3.

The purpose of this note is to give characterizations of the stability in variation in the two ways, one by constructing a Liapunov function and the other by generalizing the idea in [4].

Let $R^{n}$ be the Euclidean $n$-space with a norm $\|\cdot\|$, and set $S_{\alpha}=$ $\left\{x \in R^{n}:\|x\| \leqq \alpha\right\}$ for a given $\alpha \geqq 0$. For any matrix $A,\|A\|$ denotes the associated norm defined by $\sup _{\| x|| \leq 1}\|A x\|$.

Consider the equations

$$
\dot{x}=f(t, x),
$$

where $f(t, x)$ is continuous on $I \times R^{n}, I=[0, \infty)$, and continuously differentiable with respect to $x$. Let $x(t, s, \xi)$ be the (unique) solution of (1) through $(s, \xi)$, and put $\Phi(t, s, \xi)=(\partial / \partial \xi) x(t, s, \xi)$, which is welldefined under the assumptions. It is clear that $\Phi(t, s, \xi)$ is the fundamental matrix of the variational equations 


$$
\dot{u}=A(t, x(t, s, \xi)) u, \quad A(t, x)=(\partial / \partial x) f(t, x),
$$

and satisfies $\Phi(t, s, x(s))=\Phi(t, \tau, x(\tau)) \Phi(\tau, s, x(s)) \quad(t \geqq \tau \geqq s) \quad$ or $\quad$ more generally

$$
\Phi(t, s, x(s))=\Phi\left(t, t_{m}, x\left(t_{m}\right)\right) \Phi\left(t_{m}, t_{m-1}, x\left(t_{m-1}\right)\right) \cdots \Phi\left(t_{1}, s, x(s)\right)
$$

for any solution $x(t)$ of $(1)$ and $t \geqq t_{m} \geqq t_{m-1} \geqq \cdots \geqq t_{1} \geqq s$.

The system (1) is said to be uniformly asymptotically stable in variation, if there is a continuous function $\rho(s, \alpha)$ of $s \geqq 0, \alpha \geqq 0$ such that $\rho(s, \alpha) \rightarrow 0$ monotonously as $s \rightarrow \infty$ and that

$$
\|\Phi(t, s, \xi)\| \leqq \rho(t-s, \alpha) \quad \text { whenever } \quad \xi \in S_{\alpha} \text { and } t \geqq s .
$$

The relation (4) holds if, and only if,

$$
\|x(t, s, \xi)-x(t, s, \eta)\| \leqq \rho(t-s, \alpha)\|\xi-\eta\|, \quad \xi, \eta \in S_{\alpha},
$$

for any two solutions $x(t, s, \xi)$ and $x(t, s, \eta)$ of (1), because

$$
\begin{aligned}
x(t, s, \xi)-x(t, s, \eta) & =\int_{0}^{1}(\partial / \partial \lambda) x(t, s, \lambda \xi+(1-\lambda) \eta) d \lambda \\
& =\int_{0}^{1} \Phi(t, s, \lambda \xi+(1-\lambda) \eta) d \lambda \cdot(\xi-\eta) .
\end{aligned}
$$

Therefore, in the definition of the uniform asymptotic stability in variation, the relation (4) can be replaced by (5), and, in this case, we may drop the differentiability condition on $f(t, x)$.

REMARK 1. The uniform asymptotic stability of the system given in [10] actually requires $\|x(t, s, \xi)-x(t, s, \eta)\| \leqq \rho(t-s,\|\xi-\eta\|)$ with $\rho(t, \alpha) \rightarrow 0$ as $t \rightarrow \infty$ or as $\alpha \rightarrow 0$, which is a concept different from the stability in the above, though they pretty much resemble each other and they are coincident for linear equations.

Throughout this paper, (1) is assumed to have a bounded solution $\phi(t)$ with a bound $B_{0}$ on $I$. First of all, we shall prove the following lemmas.

Lemma 1. If the system (1) is uniformly asymptotically stable in variation, then the solutions of (1) are uniformly bounded, that is, there exists a $\beta(\alpha)$ for any $\alpha \geqq 0$ such that

$$
\|\xi\| \leqq \alpha \quad \text { implies } \quad\|x(t, s, \xi)\| \leqq \beta(\alpha) \text { for } t \geqq s .
$$

The proof is obvious since by (5) $\|x(t, s, \xi)\| \leqq\|x(t, s, \xi)-\phi(t)\|+\|\phi(t)\| \leqq \rho\left(0, \max \left\{\|\xi\|, B_{0}\right\}\right)\left(\|\xi\|+B_{0}\right)+B_{0}$.

LEMMA 2. The system (1) is uniformly asymptotically stable in 
variation if, and only if, there are positive constants $K(\alpha)$ and $c(\alpha)$ for which

$$
\|\Phi(t, s, \xi)\| \leqq K(\alpha) e^{-c(\alpha)(t-s)} \quad \text { if } \quad \xi \in S_{\alpha}, \quad t \geqq s .
$$

Proof. The sufficiency is clear. Suppose that the relation (4) holds, and choose $T(\alpha)>0$ so that $\rho(T(\alpha), \beta(\alpha))<1 / 2$, which is possible because $\rho(t, \beta(\alpha)) \rightarrow 0$ as $t \rightarrow \infty$, where $\beta(\alpha)$ is the one given in Lemma 1 . Let $\xi \in S_{\alpha}$, and set $t_{k}=s+k T(\alpha)$. If $t_{m+1}>t \geqq t_{m}$, then from (3) it follows that

$$
\begin{aligned}
\|\Phi(t, s, \xi)\| & \leqq \rho\left(t-t_{m}, \beta(\alpha)\right) \rho\left(t_{m}-t_{m-1}, \beta(\alpha)\right) \cdots \rho\left(t_{1}-s, \beta(\alpha)\right) \\
& \leqq \rho(0, \beta(\alpha)) \varepsilon^{m} \leqq\{\rho(0, \beta(\alpha)) / \varepsilon\} \varepsilon^{(t-s) / T(\alpha)} .
\end{aligned}
$$

From this, the relation (7) follows immediately by setting $K(\alpha)=$ $2 \rho(0, \beta(\alpha)), c(\alpha)=T(\alpha)^{-1} \log 2$.

q.e.d.

This lemma enables us to prove the following theorem.

THEOREM 1. Under the assumptions on $f(t, x)$, the system (1) is uniformly asymptotically stable in variation if, and only if, there exists a real-valued continuous function $W(t, x, y)$ defined on $I \times R^{n} \times R^{n}$ which satisfies

$$
\begin{gathered}
\|x-y\| \leqq W(t, x, y) \leqq M(\alpha)\|x-y\|, \\
|W(t, x, y)-W(t, u, v)| \leqq M(\alpha)\{\|x-u\|+\|y-v\|\}, \\
\dot{W}(t, x, y) \leqq-c(\alpha) W(t, x, y)
\end{gathered}
$$

on $I \times S_{\alpha} \times S_{\alpha}$ for any $\alpha \geqq 0$, where $M(\alpha)$ and $c(\alpha)$ are positive and $\dot{W}(t, x, y)=\limsup _{h \rightarrow+0}\{W(t+h, x+h f(t, x), y+h f(t, y))-W(t, x, y)\} / h$.

Proof. Necessity. Put

$$
W(t, x, y)=\sup _{s \geq t}\|x(s, t, x)-x(s, t, y)\|+\int_{t}^{\infty}\|x(s, t, x)-x(s, t, y)\| d s,
$$

which is well-defined by (5) and (7). The properties (i) and (ii) can be verified immediately. The property (iii) follows from $\dot{W}(t, x, y) \leqq$ $-\|x-y\| \leqq-W(t, x, y) / M(\alpha)$. Somewhat nontrivial is to show the continuity of $W(t, x, y)$, but it can also be proved by the standard but tedious arguments, which will be omitted.

Sufficiency. By considering $W(t, x, \phi(t))$ it is not difficult to see the uniform boundedness of the solutions of (1), that is, there is a $\beta(\alpha)$ for which (6) holds. Hence, $w(t)=W(t, x(t, s, \xi), x(t, s, \eta))$ satisfies $\dot{w}(t) \leqq$ $-c(\beta(\alpha)) w(t), w(s) \leqq M(\alpha)\|\xi-\eta\|$ if $\xi, \eta \in S_{\alpha}$, which implies (5), and hence 
(4), with $\rho(t, \alpha)=M(\alpha) \exp [-c(\beta(\alpha)) t]$.

q.e.d.

REMARK 2. Yoshizawa [10; Theorems 15.5, 21.1] has obtained a quite similar theorem about the uniform asymptotic stability of the system.

REMARK 3. Leela [6] (and Kamala-Lakshmikantham [3] for functional differential equations) constructed a Liapunov function by

$$
V(t, x)=\sup _{s \geqq t}\|x(s, t, x)\|+\int_{t}^{t+T}\|x(s, t, x)\| d s
$$

for a suitable $T>0$ under the assumption that (1) is uniformly asymptotically stable in variation and $\|x(t, s, 0)\| \leqq \lambda(s)$ for $t \geqq s$, where $\lambda(t)$ monotonously tends to 0 as $t \rightarrow \infty$. In [6] $V(t, x)$ was expected to satisfy

$$
\dot{V}(t, x) \leqq\|x(t+T, t, x)\|-\|x\|
$$

among other conditions. However, $T$ in (8) generally depends on $x$, and we cannot ignore $\partial T / \partial x$, which makes (9) uncertain.

The following theorem presents another kind of characterization of the uniform asymptotic stability in variation, which is a generalization of the idea in [4] concerning the uniform stability in variation, that is, $\|\Phi(t, s, \xi)\| \leqq \rho(\alpha)$ if $\xi \in S_{\alpha}$.

THEOREM 2. Under the assumptions on $f(t, x)$, the system (1) is uniformly asymptotically stable in variation if, and only if, the solutions of the product system

$$
\dot{x}=f(t, x), \quad \dot{\imath}=A(t, x) u,
$$

where $A(t, x)$ is the one given in (2), are uniformly bounded and uniformly ultimately bounded, that is, there are $\beta(\alpha), N(\alpha), B$ and $T(\alpha, \gamma)$, depending on the indicated arguments, such that

$$
\|x(t, s, \xi)\| \leqq \beta(\alpha), \quad\|\Phi(t, s, \xi)\| \leqq N(\alpha) \quad \text { if } \xi \in S_{\alpha}, \quad t \geqq s,
$$

and

$$
\begin{aligned}
&\|x(t, s, \xi)\| \leqq B, \quad\|\Phi(t, s, \xi) \eta\| \leqq B \\
& \text { if } \xi \in S_{\alpha}, \quad \eta \in S_{i}, \quad t \geqq s+T(\alpha, \gamma) .
\end{aligned}
$$

In the above, we note that $(x(t, s, \xi), \Phi(t, s, \xi) \eta)$ is the solution of (10) through $(s, \xi, \eta)$.

Proof. Sufficiency. By the first part of (11), the solution $x(t)=$ $x(t, s, \xi)$ of (1) satisfies $\|x(t)\| \leqq \beta(\alpha)$ if $\xi \in S_{\alpha}$ for $t \geqq s$. Hence, the 
second parts of (11) and (12) imply $\|\Phi(t, \tau, x(\tau))\| \leqq N(\beta(\alpha))$ for $t \geqq \tau \geqq s$ and $\|\Phi(t, \tau, x(\tau))\| \leqq 1 / 2$ if $t \geqq \tau+T(\beta(\alpha), 2 B)$ and $\tau \geqq s$. Thus, choosing $t_{k}=s+k T(\beta(\alpha), 2 B)$, we have

$$
\|\Phi(t, s, \xi)\| \leqq N(\beta(\alpha)) 2^{-m} \leqq 2 N(\beta(\alpha)) 2^{-(t-s) / T(\beta(\alpha), 2 B)},
$$

if $t_{m+1}>t \geqq t_{m}$, by applying (3), which proves the uniform asymptotic stability in variation.

Necessity. Suppose that (1) is uniformly asymptotically stable in variation. Then the second parts of (11) and (12) hold immediately. On the other hand, the first part of (11) is nothing but Lemma 1, while $\|x(t, s, \xi)\| \leqq\|x(t, s, \xi)-\phi(t)\|+\|\phi(t)\| \leqq 1+B_{0}$ for $t \geqq s+T(\alpha)$, if $T(\alpha)$ is chosen so that $\rho(T(\alpha), \alpha)<1 /\left(\alpha+B_{0}\right)$.

q.e.d.

COROLLARY. Suppose that there exists a continuous function $U(t, x, u)$ defined on $I \times\left\{(x, u) \in R^{n} \times R^{n}:\|x\|+\|u\| \geqq H\right\}$ for an $H>0$ which satisfies

$$
\begin{gathered}
a(\|x\|+\|u\|) \leqq U(t, x, u) \leqq b(\|x\|+\|u\|), \\
\dot{U}(t, x, u) \leqq-c(\|x\|+\|u\|),
\end{gathered}
$$

where $a(r) \rightarrow \infty$ as $r \rightarrow \infty, c(r)>0$ and

$$
\dot{U}(t, x, u)=\limsup _{h \rightarrow+0}\{U(t+h, x+h f(t, x), u+h A(t, x) u)-U(t, x, u)\} / h .
$$

Then the system (1) is uniformly asymptotically stable in variation.

Since $U(t, x, u)$ guarantees the uniform boundedness and the uniform ultimate boundedness of the solutions of (10) [10: Theorems 10.2, 10.4], the conclusion follows from Theorem 2.

ExAmple. Consider the system

$$
\dot{x}=y-F(x), \quad \dot{y}=-x-G(y),
$$

where $F(x)=\int_{0}^{x} f(s) d s, G(x)=\int_{0}^{x} g(s) d s$ with continuous functions $f(x)$, $g(x) \geqq a>0$ for a constant $a$. Then the corresponding product system is

$$
\dot{x}=y-F(x), \quad \dot{y}=-x-G(y), \quad \dot{u}=v-f(x) u, \quad \dot{v}=-u-g(y) v,
$$

and $U(t, x, y, u, v)=x^{2}+y^{2}+u^{2}+v^{2}$ satisfies the conditions required in Corollary. In fact, $\dot{U}(t, x, y, u, v) / 2=-F(x) x-G(y) y-f(x) u^{2}-g(y) v^{2} \leqq$ $-a U(t, x, y, u, v)$. Therefore, the system (13) is uniformly asymptotically stable in variation. 


\section{REFERENCES}

[1] V. M. AlekSeEv, An estimate for the perturbations of the solutions of ordinary differential equations, Vestnik Moskov. Univ. Ser. I. Mat. Meh. 2 (1961), 28-36.

[2] F. BRAUER, Perturbations of nonlinear systems of differential equations, J. Math. Anal. Appl. 14 (1966), 198-206; II, ibd. 17 (1967), 418-434; III (with A. Strauss), ibd. 31 (1970), 37-48; IV, ibd. 37 (1972), 214-222.

[3] P. S. Kamala and V. Lakshmikantham, Asymptotic self invariant sets and functional differential equations in Banach spaces, Ann. di Mat. Pura ed Appl. 92 (1972), 217-231.

[4] J. KAто, A remark on a result of Strauss, Lecture Notes in Math., Springer-Verlag, Berlin-Heidelberg-New York, 60 (1968), 83-91.

[5] J. P. LASALLE, A study of synchronous asymptotic stability, Ann. of Math. 65 (1957), 571-581.

[6] S. LeELA, Asymptotically self invariant sets and perturbed systems, Ann. di Mat. Pura ed Appl. 92 (1972), 85-93.

[7] B. G. Pachpatte, Perturbations of nonlinear systems of differential equations, J. Math. Anal. Appl. 51 (1975), 550-556.

[8] G. SeLL, Topological Dynamics and Ordinary Differential Equations, van Nostrand Reinhold Math. Studies 33, London, 1971.

[9] A. Strauss, On the stability of a perturbed nonlinear system, Proc. Amer. Math. Soc. 17 (1966), 803-807.

[10] T. Yoshizawa, Stability Theory by Liapunov's Second Method, Publ. Math. Soc. Japan 9, Tokyo, 1966.

Mathematical Institute

TOHOKU UNIVERSITY

SENDAI, 980

JAPAN

AND

FuchU-Nishi High School

FUCHU-SHI, TOKYO 183

JAPAN 